\title{
Carbon materials in Antarctic and nonAntarctic carbonaceous chondrites: high-resolution transmission electron microscopy
}

\author{
Takayuki AOKI ${ }^{*, * *}$ and Junji AKAI ${ }^{* * *}$ \\ * Graduate School of Science and Technology, Niigata University, 8050, \\ Ikarashi 2-nocho Nishi-ku Niigata 950-2181, Japan \\ ** Present address: Asahi Carbon Co. Ltd., 2 Kamomejima-machi Higashi-ku \\ Niigata 950-0883, Japan \\ *** Department of Geology, Faculty of Science, Niigata University, 8050, \\ Ikarashi 2-nocho Nishi-ku Niigata 950-2181, Japan
}

\begin{abstract}
Carbon materials from eight Antarctic carbonaceous chondrites (CM2, CO3, and CV3 types) and two nonAntarctic carbonaceous chondrites (CM2 and CV3 types) were studied using a high-resolution transmission electron microscope (HRTEM). Nanodiamonds and "carbonaceous globules" were found in seven of the Antarctic chondrites and one nonAntarctic CM2 chondrite. These results suggest that nanodiamonds and carbonaceous globules are common in carbonaceous chondrites. The finding of carbonaceous globules in the meteorites that experienced post-hydration high-temperature thermal metamorphism suggests that they are resistant to thermal metamorphism. Well-crystallized graphite was mainly found in the CV3- and CO3-type chondrites, whereas poorly crystallized graphite was mainly found in the CM2-type chondrites. The CM2-type chondrites also contain characteristic carbon structures that are similar to tetrahedral carbon onions and multilayered fullerene. In this study, we discuss the origin of these types of carbon material.
\end{abstract}

Keywords: TEM, Carbonaceous chondrite, Antarctic meteorite, Carbon material, Diamond, Graphite, Carbonaceous Globules

\section{INTRODUCTION}

A variety of fine-grained carbonaceous materials are present in the matrices of carbonaceous chondrites. The examination of these materials is the key to understanding the origin of carbonaceous materials in the early solar system.

The presence of diamond grains in carbonaceous chondrite was first reported by Lewis et al. (1987), who found diamond grains in the Allende (CV3) meteorite. Since the grains were less than $2 \mathrm{~nm}$ in diameter, they were termed as nanodiamonds (Lewis et al., 1987, 1989; Fraundorf et al., 1989; Huss, 2005). Isotope anomalies of noble gases were observed in these nanodiamonds and were interpreted to be presolar in origin (Huss and Lewis, 1995; Amari et al., 1995). On the basis of the observations

doi:10.2465/jmps.070301

T. Aoki, aokit@beige.plala.or.jp Corresponding author

J. Akai, akai@sc.niigata-u.ac.jp of twinned structures and dislocations in the diamonds, Daulton et al. (1996) proposed that nanodiamonds were formed by a chemical vapor deposition (CVD) process. On the other hand, Kimura et al. (2000) reported that diamonds could be obtained by heating quenched carbonaceous composite (QCC) in vacuum. Nakano et al. (2002) attempted to produce diamonds by aqueous alteration and subsequent thermal metamorphism of organic materials, and they suggested that the nanodiamonds developed on the meteorite parent bodies.

The presence of graphite in the Allende meteorite was confirmed by Smith and Buseck (1981) using HRTEM. Rietmeijer and Mackinnon (1985) carried out TEM observations of poorly graphitized carbon (PGC) in interplanetary dust particles (IDPs) and carbonaceous chondrites, and they suggested that the $\mathrm{d}_{002}$ spacing of PGC is related to the degree of thermal metamorphism that was experienced by the host IDPs and meteorites. Amari et al. $(1990,1995)$ suggested that a small amount of presolar 
Table 1. Meteorite samples used for TEM observation

\begin{tabular}{lccl}
\hline \multicolumn{1}{c}{ Name } & Classification & Processed Weight $(\mathrm{g})$ & \multicolumn{1}{c}{ References } \\
\hline Allende & CV3 & & \\
Murchison & CM2 & $0.44+1.44$ & \\
Asuka-882094 & CO3 & 0.31 & $1), 2), 3), 4)$ \\
Yamato-74662 & CM2 & 0.28 & $5), 6), 7)$ \\
Yamato-790992 & CO3 & 0.3 & $4), 7), 8), 9), 10), 11), 12)$ \\
Yamato-793321 & CM2 & 0.34 & $5), 6), 13)$ \\
Yamato-81020 & CO3 & 0.29 & $4), 7), 9), 10), 11), 12), 14), 15), 16), 17), 18)$ \\
Yamato-86720 & CM2 & 0.23 & $19)$ \\
Yamato-86751 & CV3 & 0.36 & $4), 7), 8), 9), 10), 11), 12), 16), 17), 20), 21), 22), 23), 24)$ \\
Belgica-7904 & CM2 or “CI” & 0.37 & \\
\hline
\end{tabular}

1) Murae et al. (1987), 2) Nagata (1979), 3) Ikeda (1983), 4) Shimoyama et al. (1991), 5) Kojima et al. (1995), 6) Shibata (1996), 7) Lipschutz et al. (1999), 8) McGarvie et al. (1987), 9) Akai (1992), 10) Miyamoto (1992), 11) Akai and Sekine (1994), 12) Nakamura (2005), 13) Sears et al. (1991), 14) Tomeoka et al. (1989), 15) Akai (1990), 16) Bischoff and Metzler (1991), 17) Ikeda (1992), 18) Ikeda et al. (1992), 19) Murakami and Ikeda (1994), 20) Tomeoka (1990), 21) Paul and Lipschutz (1990), 22) Akai (1988), 23) Kimura and Ikeda (1992), and 24) Akai (1994).

graphite $(<2 \mathrm{ppm})$ is contained in the Murchison meteorite. Hoppe et al. (1995) examined the relationship between the morphology of graphite and the isotopic anomalies of noble gases and suggested that graphite grains with smooth surfaces are presolar in origin.

Nakamura et al. (2001) found carbonaceous globules with a core-mantle structure in the Tagish Lake meteorite. Prior to this, Saito (1999) and Saito and Akai (1999) had also found material with a structure similar to the carbonaceous globules in the Ivuna (CI1) meteorite, and they described it as an undetermined carbon material. Garvie and Buseck $(2004,2006)$ observed a variety of carbonaceous globules in the Orgueil (CI1), Tagish Lake (ungrouped), and Murchison (CM2) meteorites. NakamuraMessenger et al. (2006) found characteristic isotope anomalies of nitrogen and deuterium in the carbonaceous globules found in the Tagish Lake meteorite, and they suggested that these globules developed in the cold outermost regions of protoplanetary disks.

In order to obtain more detailed information about the carbon materials, we carried out a TEM examination of eight Antarctic and two nonAntarctic carbonaceous chondrites (Table 1). Previous studies showed that three of the Antarctic carbonaceous chondrites experienced post-hydration thermal metamorphism (e.g., Akai, 1988, 1990, 1992; Tomeoka et al., 1989; Tomeoka, 1990; Ikeda, 1992; Nakamura, 2005). Hence, in this study, we examined the effect of thermal metamorphism on carbon materials.

\section{MATERIALS AND METHODS}

For this study, we examined eight Antarctic carbonaceous chondrites, which were supplied by the National Institute for Polar Research, Japan, and the Allende (CV3) and Murchison (CM2) meteorites. The following Antarctic meteorites were used: Asuka-882094 (CO3), Yamato86751 (CV3), Yamato-790992 (CO3), Yamato-81020 (CO3), Yamato-74662 (CM2), Yamato-86720 (CM2), Yamato-793321 (CM2), and Belgica-7904 (CM2 or "CI1") (Table 1).

We developed a new sample separation procedure that was based on chemical dissolution. The meteorite samples were dissolved in HF (48\% concentration). They were then processed with dilute nitric acid $(30 \%$ concentration) and aqua regia at $348 \mathrm{~K}$ for $30 \mathrm{~min}$ to remove iron sulfide, chromium sulfide, etc. The residues were collected with a centrifugal separator. They were then suspended in ethanol and dropped on microgrids coated with gold.

TEM observations were carried out using a JEM-2010 (JEOL) microscope that was operated at 200 $\mathrm{kV}$ and equipped with an energy dispersive $\mathrm{X}$-ray spectroscope (EDS; Voyager IV, Noran Instruments). For the heating experiments, we used JEM-200CX (TEM, JEOL), which was equipped with EM-SHH4 (heating holder, JEOL) and EM-SHU2 (heater control unit, JEOL). We evaporated gold and placed zinc powder on TEM microgrids for the purpose of thermal conduction and temperature calibration, respectively, in the heating experiments.

It is difficult to observe a nanodiamond in TEM images because of its low contrast, especially when it is mixed with low-crystallinity carbon materials. Thus, in order to enhance contrast, we used a method that utilized overfocusing. For example, in Figure $1 \mathrm{~b}$ taken at an overfocus of $\sim 67.2 \mathrm{~nm}$, the diamond grains can be distinguished more easily than in Figure 1a, which was taken at a normal focus. We also treated the Murchison sample 

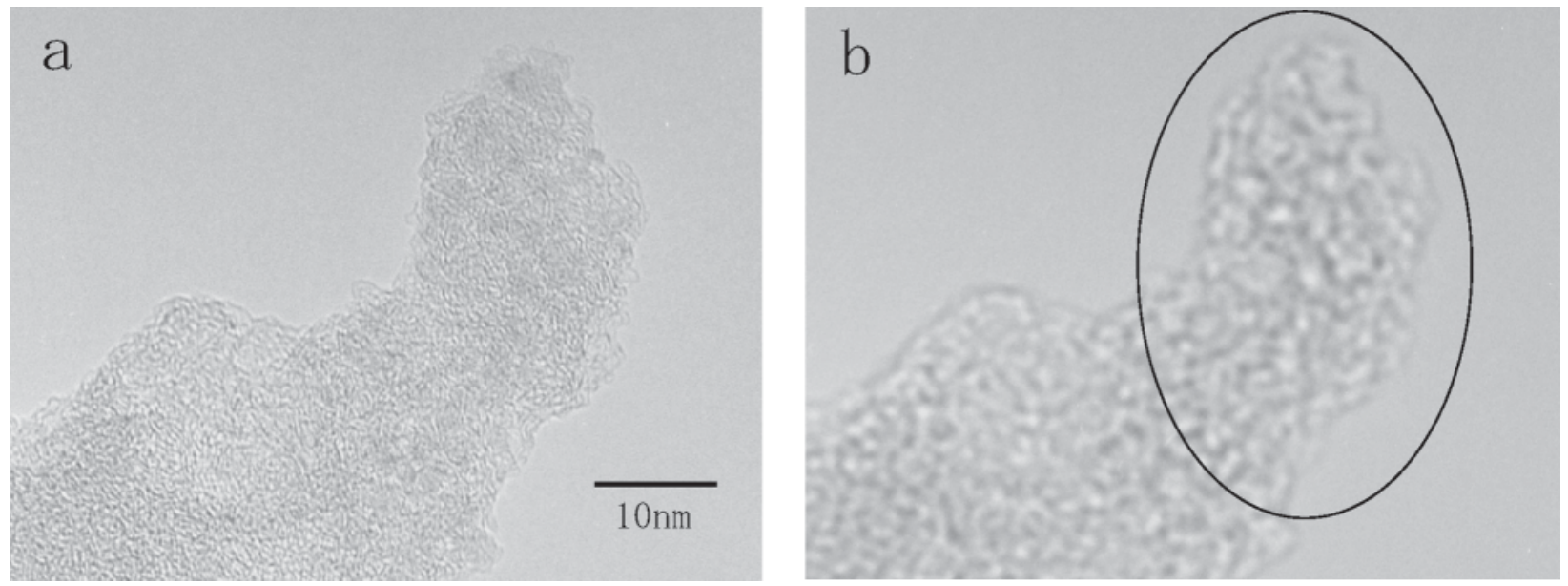

Figure 1. HRTEM images of nanodiamond grains in Yamato-74662. (a): Image with near Scherzer focus. (b): Image with overfocus. It should be noted that the nanodiamond grains (indicated by a circle) are more distinguishable in the overfocused image.

with hydrogen peroxide $\left(\mathrm{H}_{2} \mathrm{O}_{2}\right)$ to remove low-crystallinity carbon materials.

Graphite generally occurs in two polytypes-hexagonal (graphite-2H) and rhombohedral (graphite-3R). We studied many TEM simulation images of the two graphite polytypes and found that graphite $-2 \mathrm{H}$ and graphite- $3 \mathrm{R}$ can be distinguished on the basis of the intersection angle of their lattice fringes, which have a spacing of $\sim 0.34 \mathrm{~nm}$ and a spacing of $\sim 0.21 \mathrm{~nm}$, respectively (Figs. $3 \mathrm{a}$ and $3 b)$. The presence of graphite- $2 \mathrm{H}$ and graphite- $3 \mathrm{R}$ was also confirmed by using TEM simulation (MssC, JEOL) images. In the simulation images, it was observed that the (002) lattice images with a spacing of $\sim 0.34 \mathrm{~nm}$ (graphite $-2 \mathrm{H})$ or the (003) lattice images with a spacing of $0.34 \mathrm{~nm}$ (graphite-3R) showed a strong contrast, whereas the (100) lattice images with a spacing of $\sim 0.21 \mathrm{~nm}$ showed a weak contrast. We found that graphite polytypes can be determined on the basis of the intersection angle and by the change in contrast in through-focus images.

\section{RESULTS}

\section{Nanodiamonds}

Nanodiamond grains were found in the following seven Antarctic meteorites using the overfocusing method: Y-790992 (CO3), B-7904 (CM2 or CI1), Y-86751 (CV3), Y-86720 (CM2), Y-793321 (CM2), Y-81020 (CO3), Y-74662 (CM2), and Murchison (CM2). The results are summarized in Table 2. Most nanodiamond grains had a rugged spherical shape and a diameter of $\sim 3$ $\mathrm{nm}$. Figure 2a shows an aggregate of the nanodiamond grains without clear crystal faces in Belgica-7904. Figure $2 \mathrm{~b}$ shows a single-crystal nanodiamond grain in Yama-

Table 2. Summary of the TEM observations of carbon materials in the Antarctic carbonaceous chondrites

\begin{tabular}{|c|c|c|c|c|c|c|}
\hline & Class & Nano-diamond & $\begin{array}{l}\text { Well } \\
\text { crystallzed } \\
\text { graphite }\end{array}$ & $\begin{array}{l}\text { Cabonaceous } \\
\text { globules and } \\
\text { related carbon } \\
\text { materials }\end{array}$ & $\begin{array}{l}\text { Structural change } \\
\text { of low crystalline } \\
\text { carbon materials }\end{array}$ & $\begin{array}{c}\text { Heating } \\
\text { stage }\end{array}$ \\
\hline Allende & CV3 & & $\mathrm{O}$ & & O & \\
\hline A-882094 & $\mathrm{CO} 3$ & & $\bigcirc$ & & $\bigcirc$ & \\
\hline Y-790992 & $\mathrm{CO} 3$ & 0 & $\triangle$ & $\bigcirc$ & 0 & \\
\hline B-7904 & CM2 or "CI" & $\bigcirc$ & & (2) & 0 & IV \\
\hline$Y-86751$ & CV3 & $\bigcirc$ & & $\bigcirc$ & $\bigcirc$ & \\
\hline Y-86720 & $\mathrm{CM} 2$ & 0 & & (a) & 0 & IV \\
\hline Y-793321 & $\mathrm{CM} 2$ & $\bigcirc$ & & (a) & 0 & II \\
\hline Y-81020 & $\mathrm{CO} 3$ & $\bigcirc$ & & (2) & $\bigcirc$ & \\
\hline Murchison & CM2 & $\bigcirc$ & $\triangle\left(\mathrm{H}_{2} \mathrm{O}_{2}\right)$ & 0 & 0 & I \\
\hline Y-74662 & $\mathrm{CM} 2$ & 0 & & 0 & & I \\
\hline
\end{tabular}

The quantity of each material is expressed as follows:

$\triangle$, low; $\bigcirc$, medium; ( ), high.

The degree of heating increases in stages as follows: I $<$ II $<$ III $<$ IV.

The notification used for the heating stages corresponds to those used by Nakamura (2005). 

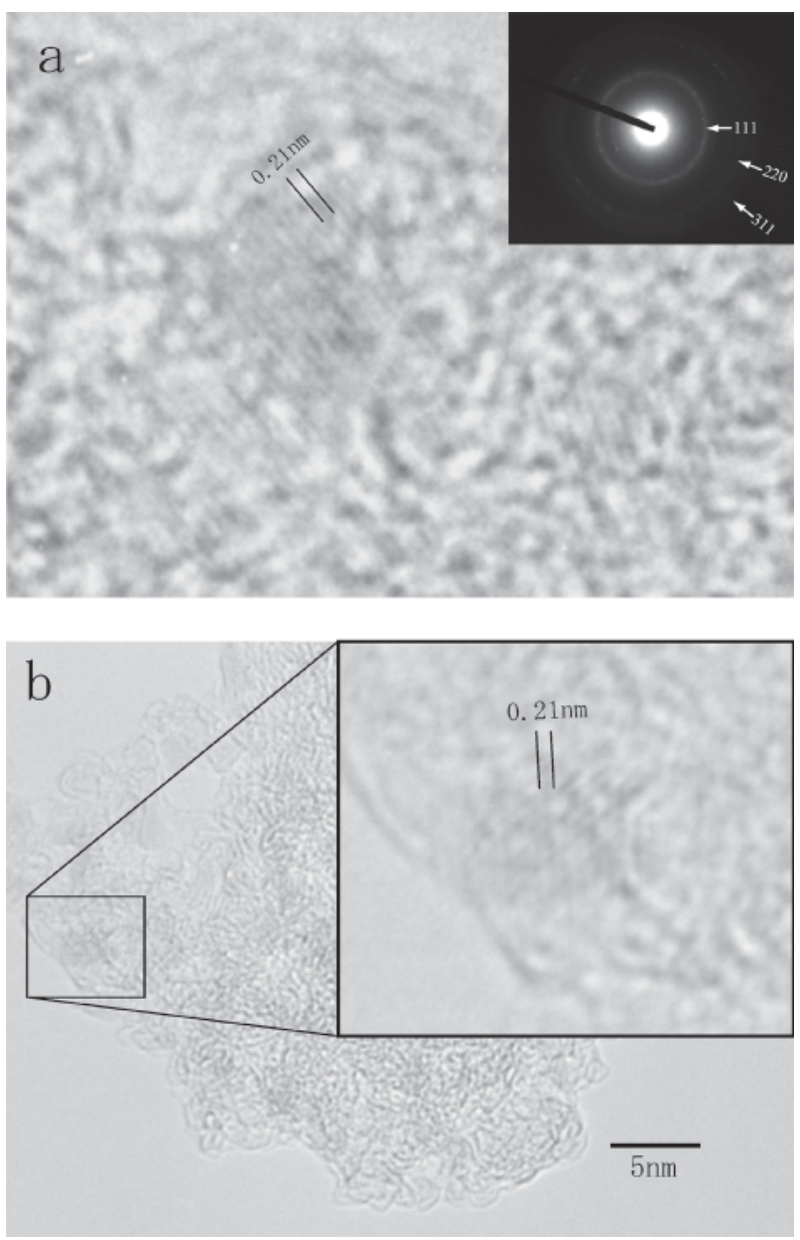

Figure 2. (a): HRTEM image of aggregated nanodiamond grains in Belgica-7904. Image of a diamond with a (111) lattice having a spacing of $0.21 \mathrm{~nm}$. In the inset is an ED pattern of the nanodiamond grains. (b): HRTEM image of a single-crystal nanodiamond grain in $\mathrm{Y}-86720$.

to-86720. No significant differences were observed in the amount of nanodiamond grains present in the meteorites.

\section{Graphite and poorly crystallized carbon materials}

Well-crystallized graphite. Thick, platy well-crystallized graphite was found in the Allende (CV3), A-882094 (CO3), Y-790992 (CO3), and Murchison (CM2, treated with $\mathrm{H}_{2} \mathrm{O}_{2}$ ) meteorites. Lattice fringes with an interlayer spacing of $\sim 0.34 \mathrm{~nm}$ were observed in the graphite. Moreover, the diffraction rings in the electron diffraction (ED) patterns were very sharp (Figs. 3a and 3b). We determined the particular polytype of graphite using the method described in the Materials and Methods section. Our observations revealed that the presence of graphite$2 \mathrm{H}$ was predominant and that of graphite- $3 \mathrm{R}$ was comparatively rare in the meteorites.

Ribbon-film-like carbonaceous material. We found rib-
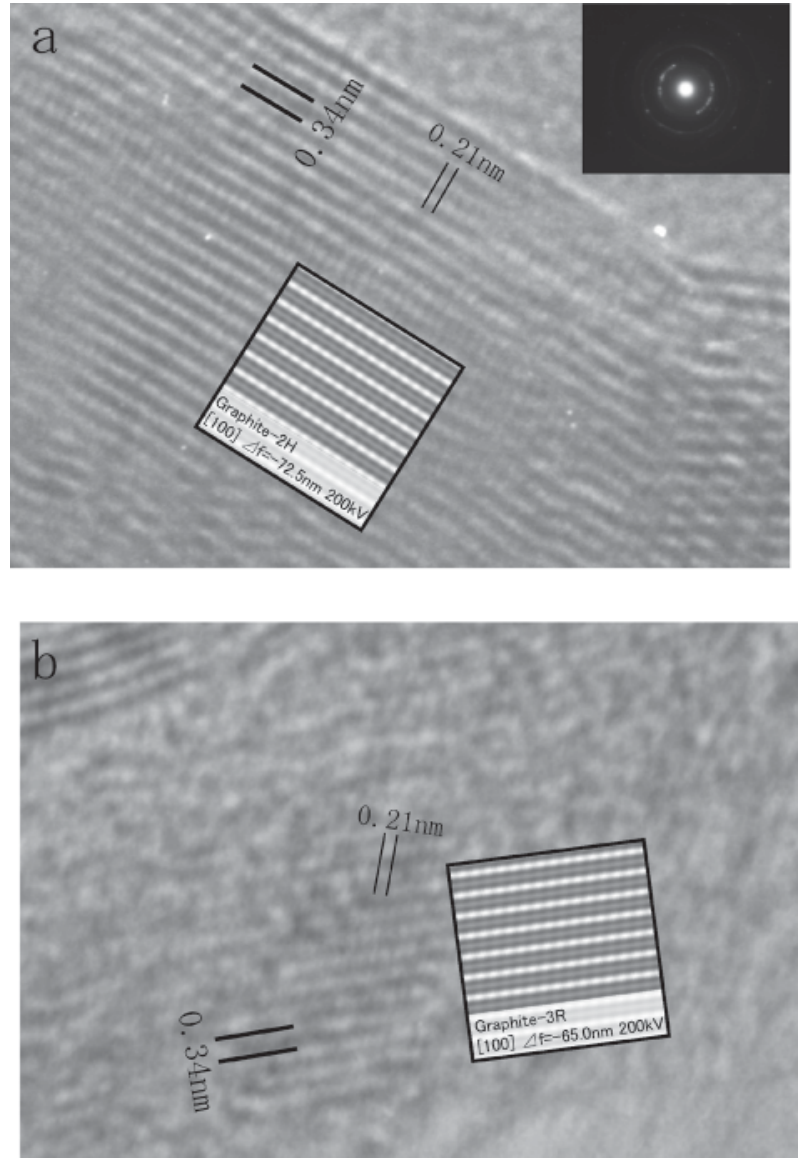

Figure 3. HRTEM images of graphite grains in Asuka-882094. (a): Image of a thick platy well-crystallized graphite grain. In the inset is its ED pattern. It can be observed that the fringes with a spacing of $0.21 \mathrm{~nm}$ are orthogonal to those with a spacing of 0.34 $\mathrm{nm}$. The lattice configuration corresponds to graphite-2H. (b): Image of a graphite-3R grain. It can be observed that the fringes with a spacing of $0.21 \mathrm{~nm}$ diagonally intersect those with a spacing of $0.34 \mathrm{~nm}$.

bon-film-like carbonaceous material in almost all the meteorites that were studied. Their rims were generally thick, and they had a complicated network structure of the order of micrometers (Fig. 4). We observed that as the degree of crystallization in the material increased, the lattice layers with a spacing of $0.34 \mathrm{~nm}$ became pronounced along the grain edge, suggesting that their presence was related to that of graphite.

Spherical carbonaceous globules. Spherical carbonaceous globules, typically $50-300 \mathrm{~nm}$ in diameter, were found to characteristically occur in the Murchison and other CM-type chondrites. The globules had a thick balllike structure (Fig. 5). Their ED patterns showed only halos, indicating that they were largely amorphous. An EDS analysis showed that the globules contained $\mathrm{S}$ and $\mathrm{Cl}$, in addition to $\mathrm{C}$.

Concentric-sphere-type carbon material. Concentric 


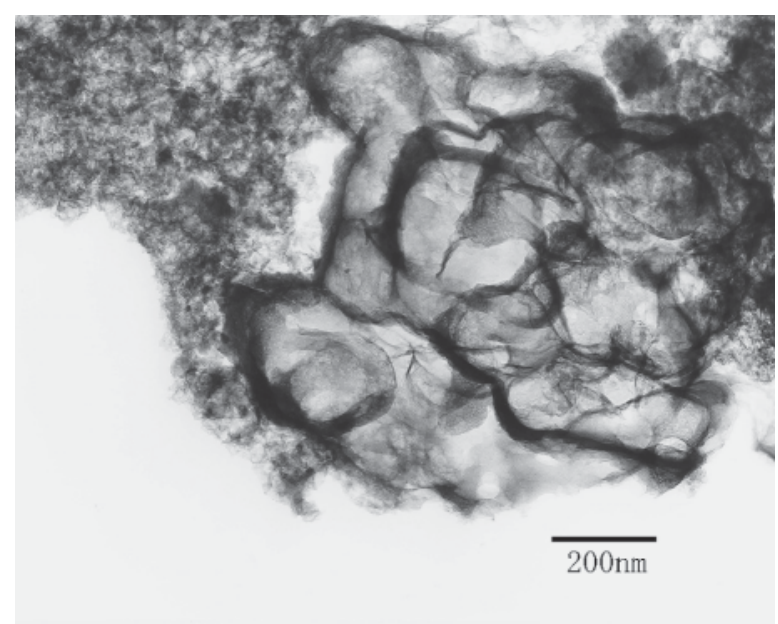

Figure 4. TEM image of ribbon-film-like carbonaceous material in B-7904.

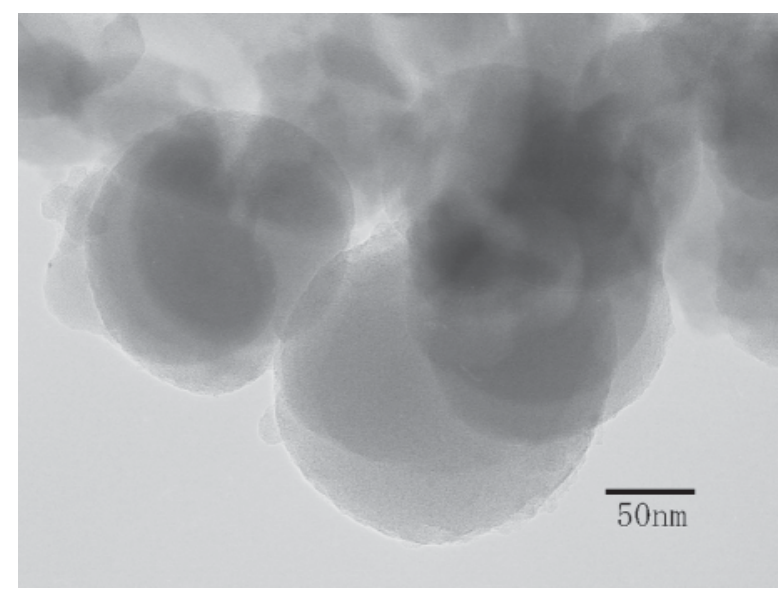

Figure 5. TEM image of spherical carbonaceous globules in Murchison. Their ED pattern shows only a halo, indicating that they are amorphous.

sphere-type carbon material grains with a finger print or onion-like structure were found in Belgica-7904. Lattices were observed even in the central part of the carbon material (Fig. 6). This carbon material may have a genetic relationship to the spherical carbon globules described above.

Featureless carbon material. Featureless carbon material was found to generally occur in the CM2 meteorites. This material showed only a halo in the ED patterns, indicating that it was largely amorphous. However, very small crystalline lattices with a spacing of $\sim 0.34 \mathrm{~nm}$ were sometimes found in these meteorites. This type of carbon material also contained small amounts of $\mathrm{S}$ and $\mathrm{Cl}$.

\section{Carbon onions}

Characteristic onion-like nanocarbon material with 1 to 4

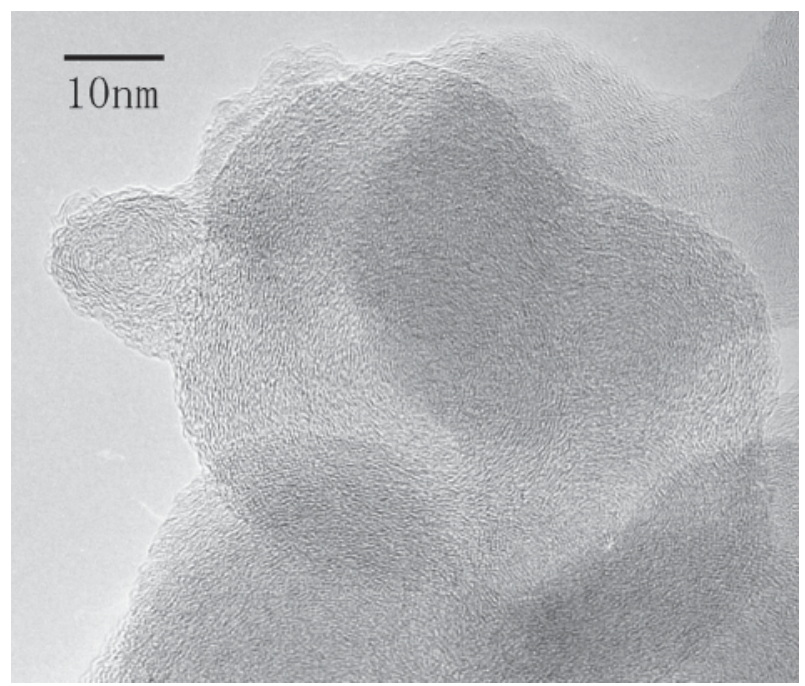

Figure 6. TEM image of concentric-sphere-type carbon material in Belgica-7904. This material has a structure similar to fingerprint-like or onion-like patterns. Lattice fringes can be seen even in its central part. The concentric-sphere-type carbon material may have a genetic relationship to the carbonaceous globules.

layers, which ranged in diameter from $0.8-3 \mathrm{~nm}$, was observed in the Murchison sample treated with $\mathrm{H}_{2} \mathrm{O}_{2}$ (Figs. $7 \mathrm{a}$ and $7 \mathrm{~b}$ ). The material shown in Figure $7 \mathrm{a}$ was similar to the tetrahedral carbon onions reported by Oku et al. (2004). Figure $7 \mathrm{~b}$ shows the carbon material that corresponds to the multilayered fullerene-like structure reported by Dodziuk et al. (2000). Such nanocarbon onions were not observed in any other meteorites.

\section{Carbonaceous globules}

Carbonaceous globules were observed in the following seven Antarctic meteorites: Yamato-793321 (CM2), Y-81020 (CO3), Y-86720 (CM2), Y-86751 (CV3), Y-790992(CO3), Y-74662 (CM2), Belgica-7904 (CM2 of CI1), and Murchison (CM2). Figures $8 \mathrm{a}-\mathrm{f}$ show the typical TEM images of the carbonaceous globules. The globules in all the meteorites were of a similar size and shape and had a nearly identical composition with minor amounts of $\mathrm{O}, \mathrm{S}$, and $\mathrm{Cl}$. They were found to be largely amorphous and had a core-mantle structure. Their diameters ranged from $200-700 \mathrm{~nm}$. These globules probably correspond to those described in the Tagish Lake meteorite (Nakamura et al., 2002).

We carried out in situ heating experiments of carbonaceous globules using an electron microscope in order to examine their thermal stability. Figures $9 \mathrm{a}$ and $9 \mathrm{~b}$ show a carbonaceous globule before and after it was heated to $773 \mathrm{~K}$, respectively. No significant changes were observed in either the amount of graphitization or in the shape and 

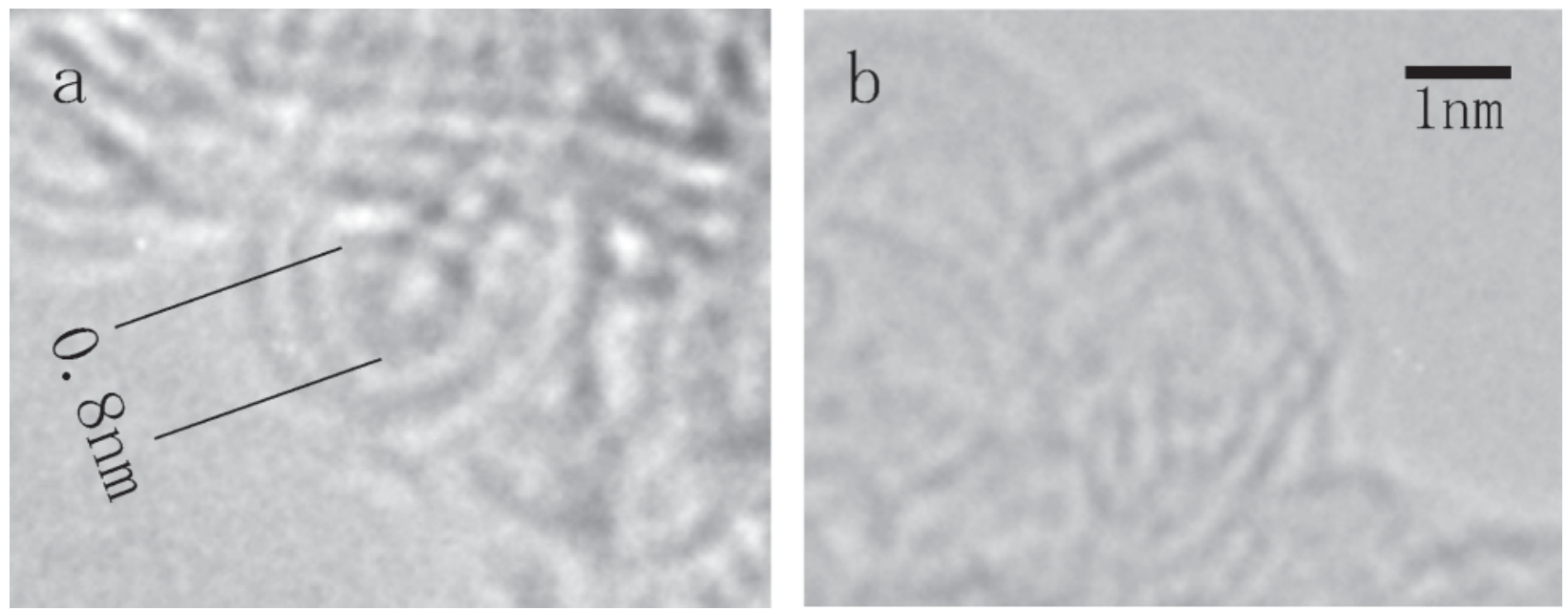

Figure 7. (a): HRTEM image of a characteristic carbon material similar to a tetrahedral carbon onion. (b): HRTEM image of a carbon material exhibiting multilayered fullerene-like structure. Both images are of material obtained from Murchison.

size of the globules. The ED patterns showed that the globule remained amorphous even after heating.

\section{DISCUSSION}

\section{Nanodiamonds}

Nanodiamond grains were found in seven of the Antarctic meteorites and in the Murchison meteorite. Nanodiamond grains were clearly more abundant than well-crystallized graphite grains. This result appears to be consistent with the theoretical interpretation by Badziag et al. (1990) that nanodiamond grains smaller than $3 \mathrm{~nm}$ in diameter are energetically favored over polycyclic aromatic hydrocarbons (precursors to graphite) when the surface bonds are terminated with hydrogen.

A presolar CVD formation model has been widely accepted as the theory of the origin of nanodiamonds (e.g., Dalton et al., 1996; Henning and Salama, 1998; Huss, 2005). However, recently, another hypothesis has been proposed, which states that nanodiamonds were formed in the inner solar nebula (Dai et al., 2002; Kouchi et al., 2005). On the basis of the results of hydrothermal experiments, Kouchi et al. (2005) suggested that nanodiamonds were formed on the meteorite parent bodies by aqueous alteration and subsequent thermal metamorphism. However, their TEM identification of nanodiamonds needs to be more clearly verified, and their model for the formation of the nanodiamonds under low-pressure aqueous alteration is still debatable. Our study revealed that nanodiamond grains occur even in thermally unmetamorphosed carbonaceous chondrites, which implies that the theory of presolar origin is more probable than that of parent-body origin.

\section{Carbonaceous globules}

Carbonaceous globules were also observed in seven of the Antarctic meteorites and in the Murchison meteorite. The results suggest that carbonaceous globules are very common in carbonaceous chondrites, which is consistent with the conclusion of Garvie (2006). Carbonaceous globules may be considered as solid organic matter because they contain considerable amounts of oxygen, sulfur, and chlorine. Their compositions are similar to the globules in the Tagish Lake carbonaceous chondrite (Nakamura et al., 2001, 2002). As suggested by Nakamura-Messenger et al. (2006), these globules may be primitive carbon material formed in the cold outermost regions of protoplanetary disks.

It is speculated that carbonaceous globules may have some importance in relation to the origin of life on Earth. The carbonaceous globules found in the meteorites are similar in size to the smallest bacteria in recent Earth environments. If carbonaceous globules were present on the surface of Earth in the early stage of its evolution, it is possible that they played some role in the birth and/or early evolution of life on Earth.

\section{Graphite and other varieties of carbon materials}

Platy well-crystallized graphite grains were found to be abundant in the Allende (CV3), A-882094 (CO3), Y790992 (CO3), and Murchison (CM2, treated with $\mathrm{H}_{2} \mathrm{O}_{2}$ ) meteorites. In comparison, well-crystallized graphite was relatively uncommon in the $\mathrm{CM} 2$ meteorites.

Based on experiments of graphite formation from organic matters, Kouchi et al. (2005) suggested that graphite may have formed on the meteorite parent bodies during 

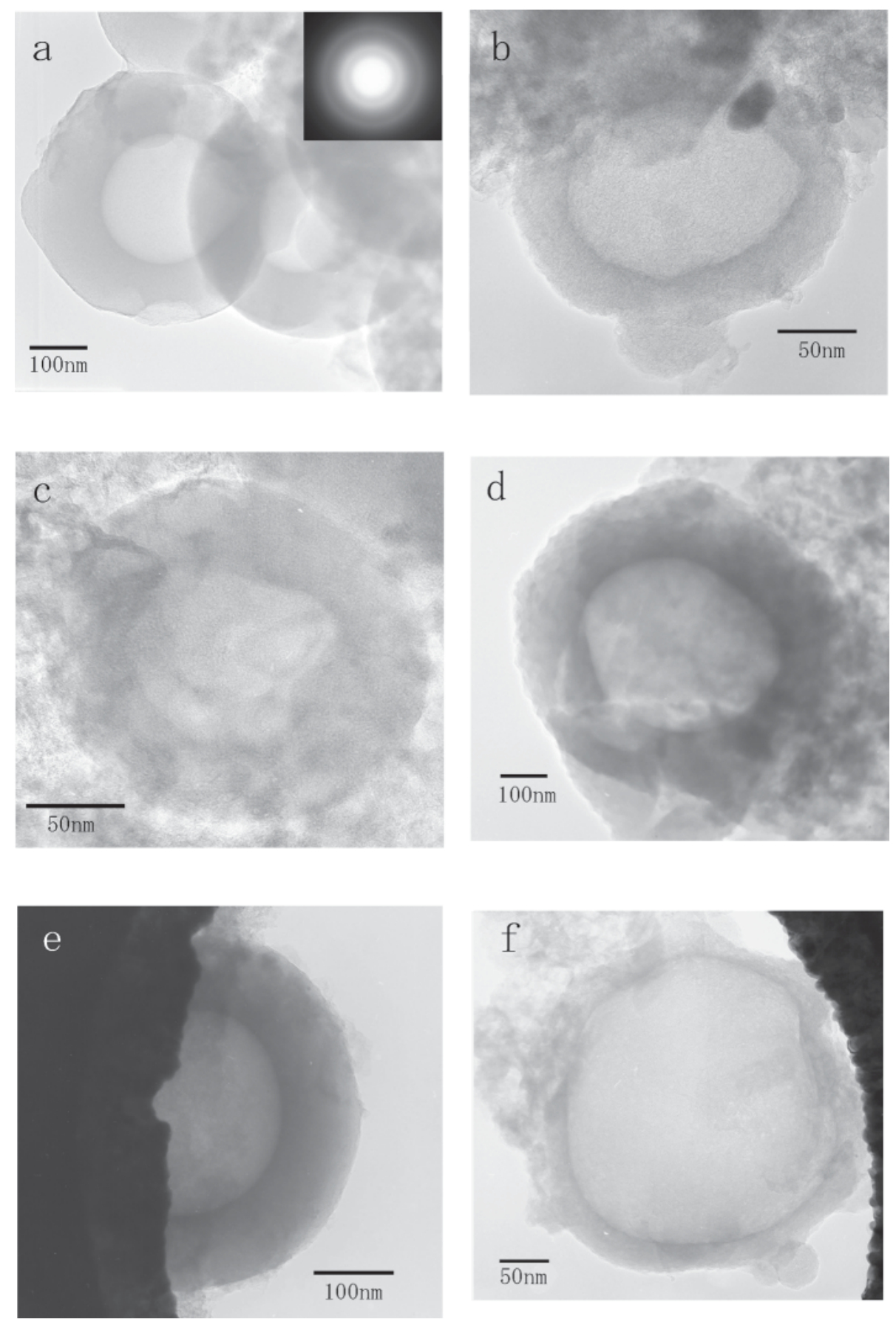

Figure 8. TEM images of carbonaceous globules in Antarctic meteorites. (a): Belgica-7904, (b): Yamato-790992, (c): Y-86751, (d): Y-74662, (e): Y-81020, and (f): Y-793321.

thermal metamorphism. However, our study showed that a small amount of graphite was present in Murchison (CM2), which did not undergo post-hydration thermal metamorphism. Thus, the formation of platy well-crystallized graphite need not necessarily be ascribed to thermal metamorphism.

Ribbon-like and film-like carbonaceous grains were found in almost all the meteorites, suggesting that these types of grains may be a stable and common form of carbonaceous material. Graphite lattice layers with a spacing of $\sim 0.34 \mathrm{~nm}$ were also sometimes found around the margin of the grains; this implies that the graphite layers may have developed from the low-crystallinity materials during thermal metamorphism. This occurrence was more common in samples that experienced high-temperature thermal metamorphism.

Garvie and Buseck (2006) chemically treated an Orgueil (CI1) meteorite sample and found various types of carbonaceous materials in the acid residues. The results of our study indicate that similar types of carbonaceous 

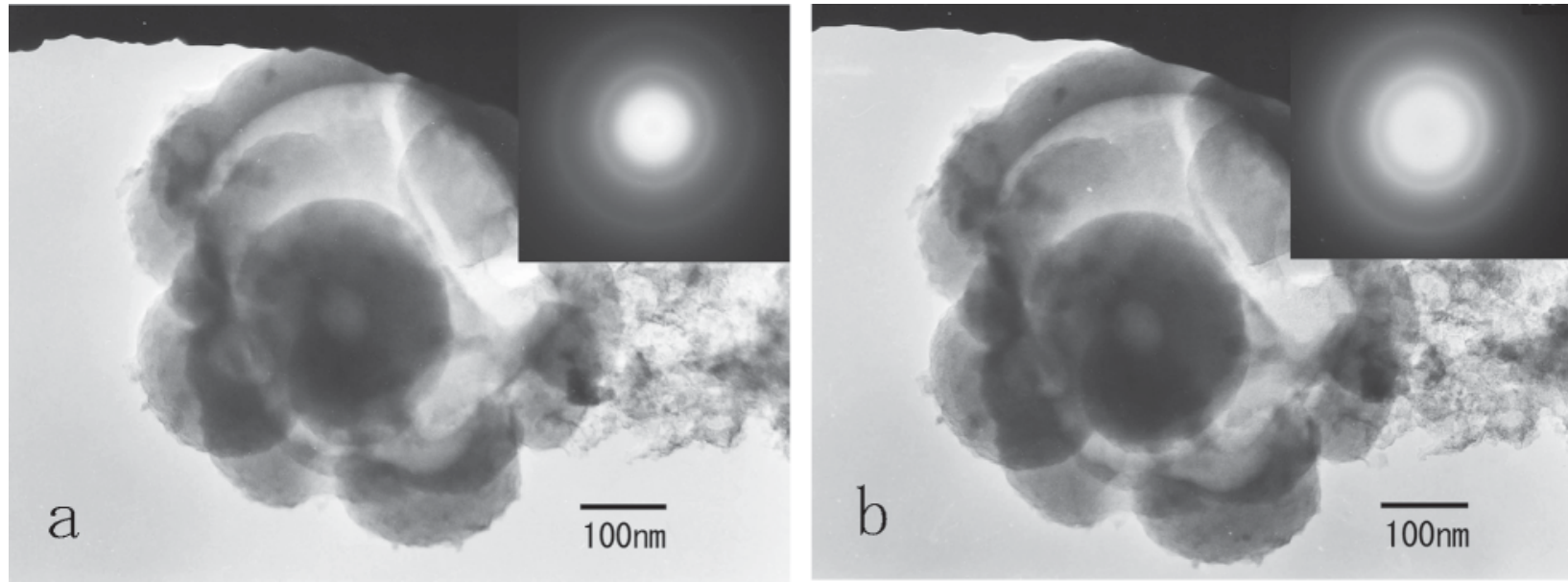

Figure 9. TEM images of carbonaceous globules in Y-86720 (a): before heating and (b): after heating to $773 \mathrm{~K}$ for 90 min in the heating experiment. No changes were observed in the characteristics.

materials occur in other carbonaceous chondrites.

Oku et al. (2004) experimentally showed that carbon onion structures develop from amorphous carbon black by strong irradiation of an electron beam during TEM observation. However, such a transformation was not observed in our study because we used a relatively broad electron beam for TEM observation and did not take images by long exposure of the electron beam. Thus, it can be inferred that the carbon onion is probably indigenous to the meteorites.

\section{Carbon materials in relation to thermal metamor- phism}

Based on the crystallinity of carbon materials, we can divide the carbonaceous chondrites into two groups: those with well-crystallized carbon materials and those with poorly crystallized carbon materials: Allende (CV3) and A-882094 (CO3) can be classified into the well-crystallized carbon materials group, while Y-81020 (CO3), B-7904 (CM2 or CI1), Y-86720 (CM2), Y-793321 (CM2), Murchison (CM2), and Y-74662 (CM2) can be classified into the poorly crystallized carbon materials group. Y-790992 (CO3) and Y-86751 (CV3) can probably be classified as being intermediate between these two groups. The grouping leads us to the suggestion that wellcrystallized carbon materials are abundant in CV3- and CO3-type chondrites, whereas poorly crystallized carbon materials are abundant in CM2-type chondrites.

Our study revealed that the carbon materials in carbonaceous chondrites exhibit a large range of structural variations. There is a tendency that the number of lattice fringes with an interlayer spacing of $0.34 \mathrm{~nm}$ increases with the increase in the degree of the thermal metamor- phism of the host meteorite. Thus, the crystallinity of the carbon materials can be a useful indicator to deduce the degree of thermal metamorphism.

However, the crystallinity of graphite may not be directly related to thermal metamorphism. Previous studies (e.g., Akai, 1988) indicated that the degree of thermal metamorphism of B-7904 was larger than that of Allende. Nonetheless, we have found that well-crystallized graphite is common in Allende, whereas it is rare in B-7904. The result suggests that platy well-crystallized graphite was formed before being accumulated on the meteorite parent body rather than during thermal metamorphism.

Nanodiamond grains were found in seven of the Antarctic meteorites, which included those that experienced strong thermal metamorphism, such as B-7904 and Y-86720. This observation suggests that these nanodiamond grains can survive strong thermal metamorphism.

\section{ACKNOWLEDGMENTS}

We are grateful to Prof. K. Tomeoka of Kobe University for reviewing our manuscript and giving us useful advice and encouragement. We also wish to thank Prof. T. Yoshimura and Dr. Andrew Whitaker of Niigata University and Dr. Y. Kawachi for reviewing our manuscript as well as Dr. T. Noguchi of Ibaraki University for his helpful comments on carbon materials. We also wish to acknowledge Dr. K. Nakamura-Messenger of NASA's Johnson Space Center and Prof. S. Nakashima of Osaka University for their constructive reviews and helpful comments. 


\section{REFERENCES}

Akai, J. (1988) Incompletely transformed serpentine-type phyllosilicates in the matrix of Antarctic CM chondrites. Geochimica et Cosmochimica Acta, 52, 1593-1599.

Akai, J. (1990) Mineralogical evidence of heating events in Antarctic carbonaceous chondrites Y-86720 and Y-82162. Proceedings of the NIPR Symposium of Antarctic Meteorites, 3, National Institute of Polar Research, Tokyo, 55-68.

Akai, J. (1992) T-T-T diagram of serpentine and saponite, and estimation of metamorphic heating degree of Antarctic carbonaceous chondrites. Proceedings of the NIPR Symposium of Antarctic Meteorites, 5, National Institute of Polar Research, Tokyo, 120-135.

Akai, J. (1994) Void structures in olivine grains in thermally metamorphosed Antarctic carbonaceous chondrite B-7904. Proceedings of the NIPR Symposium of Antarctic Meteorites, 7 , National Institute of Polar Research, Tokyo, 94-100.

Akai, J. and Sekine, T. (1994) Shock effects experiments on serpentine and thermal metamorphic conditions in Antarctic carbonaceous chondrite. Proceedings of the NIPR Symposium of Antarctic Meteorites, 7, National Institute of Polar Research, Tokyo, 101-109.

Amari, S., Anders E., Virag, A. and Zinner, E. (1990) Interstellar graphite in meteorites. Nature, 345, 238-240.

Amari, S., Lewis, R.S. and Anders, E. (1995) Interstellar grains in meteorites: III. Graphite and its noble gases. Geochimica et Cosmochimica Acta, 59, 1411-1426.

Badziag, P., Verwoerd, W.S., Ellis, W.P. and Greiner, N.R. (1990) Nanometre-sized diamonds are more stable than graphite. Nature, 343, 244-245.

Bischoff, A. and Metzler, K. (1991) Mineralogy and petrography of Y-86720, Y-82162, and B-7904. Proceedings of the NIPR Symposium of Antarctic Meteorites, 4, National Institute of Polar Research, Tokyo, 226-246.

Dai, Z.R., Bradley, J.P., Joswiak, D.J., Brownlee, D.E., Hill, H. G.M. and Genge, M.J. (2002) Possible in situ formation of meteoritic nanodiamonds in the early Solar System. Nature, 418, 157-159.

Daulton, T.L., Eisenhour, D.D., Bernatowicz, T.J., Lewis, R.S. and Buseck, P.R. (1996) Genesis of presolar diamonds: Comparative high-resolution transmission electron microscopy study of meteoritic and terrestrial nano-diamonds. Geochimica et Cosmochimica Acta, 60, 4853-4872.

Dodziuk, H., Dolgonos, G. and Lukin, O. (2000) Ease of formation of nested fullerenes. Chemical Physics Letters, 329, 351-356.

Garvie, L.A.J. (2006) Extraterrestrial carbon nanospheres. Carbon, $44,158-160$

Garvie, L.A.J. and Buseck, P.R. (2004) Nanosized carbon-rich grains in carbonaceous chondrite meteorites. Earth and Planetary Science Letters, 224, 431-439.

Garvie, L.A.J. and Buseck, P.R. (2006) Carbonaceous materials in the acid residue from the Orgueil carbonaceous chondrite meteorite. Meteoritics \& Planetary Science, 41, 633-642

Fraundorf, P., Fraundorf, G., Bernatowicz, T., Lewis, R. and Tang, M. (1989) Stardust in the TEM. Ultramicroscopy, 27, 401411

Henning, Th. and Salama, F. (1998) Carbon in the Universe. Science, 282, 2204-2210.

Hoppe, P., Amari, S., Zinner, E. and Lewis, R.S. (1995) Isotopic compositions of $\mathrm{C}, \mathrm{N}, \mathrm{O}, \mathrm{Mg}$, and $\mathrm{Si}$, trace element abundances, and morphologies of single circumstellar graphite grains in four density fractions from the Murchison meteorite. Geohimica et Cosmochimica Acta, 59, 4029-4056.

Huss, G.R. (2005) Meteoritic nanodiamonds: Messengers from the stars. Elements, 1,97-100.

Huss, G.R. and Lewis, R.S. (1995) Presolar diamond, SiC, and graphite in primitive chondrites: Abundances as a function of meteorite class and petrologic type. Geochimica et Cosmochimica Acta, 59, 115-160.

Ikeda, Y. (1983) Alteration of chondrules and matrices in the four Antarctic carbonaceous chondrites ALH-77307(C3), Y-790123(C2), Y-75293(C2) and Y-74662(C2). Memoirs of National Institute of Polar Research Special Issue, 30, National Institute of Polar Research, Tokyo, 93-108.

Ikeda, Y. (1992) An overview of the research consortium, "Antarctic carbonaceous chondrites with CI affinities, Yamato-86720, Yamato-82162, and Belgica-7904". Proceedings of the NIPR Symposium of Antarctic Meteorites, 5, National Institute of Polar Research, Tokyo, 49-73.

Ikeda, Y., Noguchi, T. and Kimura, M. (1992) Petrology and mineralogy of the Yamato-86720 carbonaceous chondrite. Proceedings of the NIPR Symposium of Antarctic Meteorites, 5, National Institute of Polar Research, Tokyo, 136-154.

Kimura, M. and Ikeda, Y. (1992) Mineralogy and petrology of an unusual B-7904 carbonaceous chondrite: genetic relationships among the components. Proceedings of the NIPR Symposium of Antarctic Meteorites, 5, National Institute of Polar Research, Tokyo, 74-119.

Kimura, S., Kaito, C. and Wada, S. (2000) Formation of micro-diamond by heat treatment of quenched carbonaceous composite (QCC). Antarctic Meteorite Research, 13, 145-152.

Kojima, T., Yada, S. and Tomeoka, K. (1995) Ca-Al-rich inclusions in three Antarctic CO3 chondrites, Yamato-81020, Yamato-82050 and Yamato-790992: Record of low-temperature alteration. Proceedings of the NIPR Symposium of Antarctic Meteorites, 8, National Institute of Polar Research, Tokyo, 79-96.

Kouchi, A., Nakano, H., Kimura Y. and Kaito, C. (2005) Novel routes for diamond formation in interstellar ices and meteoritic parent bodies. The Astrophysical Journal, 626, L129L132.

Lewis, R.S., Tang, M., Wacker, J.F., Anders, E. and Steel, E. (1987) Interstellar diamonds in meteorites. Nature, 326, 160162.

Lewis, R.S., Anders, E. and Draine, B.T. (1989) Properties, delectability and origin of interstellar diamonds in meteorites. Nature, 339, 117-121.

Lipschutz, M.E., Zolensky, M.E. and Bell, M.S. (1999) New petrographic and trace element data on thermally metamorphosed carbonaceous chondrites. Antarctic Meteorite Research, 12, 57-80.

McGarvie, D.W., Wright, I.P., Grady, M.M., Pillinger, C.T. and Gibson, Jr., E.K. (1987) A stable carbon isotopic study of types 1 and 2 carbonaceous chondrites. Memoirs of National Institute of Polar Research Special Issue, 46, National Institute of Polar Research, Tokyo, 179-195.

Miyamoto, M. (1992) Infrared diffuse reflectance spectra of several thermally metamorphosed carbonaceous chondrites. Proceedings of the NIPR Symposium of Antarctic Meteorites, 5, National Institute of Polar Research, Tokyo, 155-164.

Murae, T., Masuda, A. and Takahashi, T. (1987) Pyrolytic studies 
of carbonaceous matter in Antarctic carbonaceous chondrites. Memoirs of National Institute of Polar Research Special Issue, 46, National Institute of Polar Research, Tokyo,196-204.

Murakami, T. and Ikeda, Y. (1994) Petrology and mineralogy of the Yamato-86751 CV3 chondrite. Meteoritics, 29, 397-409.

Nagata, T. (1979) Magnetic classification of Antarctic stony meteorites (III). Proceedings of the third symposium on Antarctic meteorites National institute of Polar Resarch, National Institute of Polar Research, Tokyo, 223-239.

Nakamura, K., Zolensky, M.E., Tomita, S. and Tomeoka, K. (2001) In situ observation of carbonaceous globules in the Tagish Lake chondrite. Meteoritics \& Planetary Science, 36, A145.

Nakamura, K., Zolensky, M.E., Tomita, S., Nakashima, S. and Tomeoka, K. (2002) Hollow organic globules in the Tagish Lake meteorite as possible products of primitive organic reactions. International Journal of Astrobiology, 1 (3), 179-189.

Nakamura-Messenger, K., Messenger, S., Keller, L.P., Clemett, S.J. and Zolensky, M.E. (2006) Organic Globules in the Tagish Lake Meteorite: Remnants of the Protosolar Disk. Science, 314, 1439-1442.

Nakamura, T. (2005) Post-hydration thermal metamorphism of carbonaceous chondrites. Journal of Mineralogical and Petrological Sciences, 100, 260-272.

Nakano, H., Kouchi, A., Arakawa, M., Kimura, Y., Kaito, C., Ohno, H. and Hondoh, T. (2002) Alteration of interstellar organic materials in meteorites' parent bodies: a novel route for diamond formation. Proceedings of the Japan Academy, 78, Ser.B, 277-281.

Oku, T., Narita, I. and Nishiwaki, A. (2004) Formation, atomic structural optimization and electronic structures of tetrahedral carbon onion. Diamond and Related Materials, 13, 13371341.

Paul, R.L. and Lipshutz, M.E. (1990) Consortium study of labile trace elements in some Antarctic carbonaceous chondrites: Antarctic and non-Antarctic meteorite comparisons. Proceedings of the NIPR Symposium of Antarctic Meteorites, 3, National Institute of Polar Research, Tokyo, 80-95.

Rietmeijer, F.J.M. and Mackinnon, I.D.R. (1985) Poorly graphitized carbon as a new cosmothermometer for primitive extra- terrestrial materials. Nature, $315,733-736$

Saito, M. (1999) Electron Microscopy Mineralogy of Carbonaceous Chondrite. MS .thesis, Niigata University, Japan (in Japanese).

Saito, M. and Akai, J. (1999) Electron microscope mineralogy of magnetite from CIlchondrite, Ivna and Orgueil. Abstracts of Annual Meeting of the Mineralogical Society of Japan, 99 (in Japanese).

Sears, D.W. G., Batchelor, J.D., Lu, J. and Keck, B.D. (1991) Metamorphism of $\mathrm{CO}$ chondrites and CO-like chondrites and comparison with type 3 ordinary chondrites. Proceedings of the NIPR Symposium of Antarctic Meteorites, 4, National Institute of Polar Research, Tokyo, 319-343.

Shibata, Y. (1996) Opaque minerals in Antarctic CO3 carbonaceous chondrites, Yamato-74135, -790992, -791717, -81020, $-81025,-82050$ and Allan Hills 77307. Proceedings of the NIPR Symposium of Antarctic Meteorites, 9, National Institute of Polar Research, Tokyo, 79-96.

Shimoyama, A., Komiya, M. and Harada, K. (1991) Release of organic compounds from some Antarctic CI and CM chondrites by laboratory heating. Proceedings of the NIPR Symposium of Antarctic Meteorites, 4, National Institute of Polar Research, Tokyo, 247-260.

Smith, P.P.K. and Buseck, P.R. (1981) Graphitic carbon in the Allende meteorite: A microstructural study. Science, 212, 322-324

Tomeoka, K. (1990) Mineralogy and petrology of Belgica-7904: A new kind of carbonaceous chondrite from Antarctica. Proceedings of the NIPR Symposium of Antarctic Meteorites, 3, National Institute of Polar Research, Tokyo, 40-54.

Tomeoka, K., Kojima, H. and Yanai, K. (1989) Yamato-86720: a $\mathrm{CM}$ carbonaceous chondrite having experienced aqueous alteration and thermal metamorphism. Proceedings of the NIPR Symposium of Antarctic Meteorites, 2, National Institute of Polar Research, Tokyo, 55-74.

Manuscript received March 1, 2007

Manuscript accepted January 31, 2008

Manuscript handled by Kazushige Tomeoka 